\title{
Effects of Context on Processing (non)-Compositional Expressions
}

\author{
Edward Holsinger \& Elsi Kaiser
}

\author{
Department of Linguistics, University of Southern California, Los Angeles, USA \\ \{ holsinge, emkaiser \} dusc.edu
}

\section{Abstract}

We report a self-paced reading study investigating how contextual biases influence the processing of phrasal verbs that are ambiguous between literal and non-literal interpretations (wait on a bench, a customer $\}$ ). Our results suggest an asymmetry in how contextual bias affects processing: When context biases the non-literal interpretation, comprehension proceeds smoothly regardless of whether the verb sequence turns out to be literal or non-literal. But when context biases the literal interpretation, processing difficulties arise when the verb sequence turns out to be non-literal. We relate these findings to existing models of non-compositional processing.

\section{Introduction}

The contents and structure of the mental lexicon is central to any theory of language processing. The contents of this lexicon are widely assumed to be the individual words that an individual knows, as well as any further morphemes required for interpreting his native language. (e.g. Forester, 1976; Swinney \& Cutler, 1979; Dell, 1986).

This system interfaces with a separate system, responsible for grammatical computation, that specifies how words can be combined to create sentences (e.g. Levelt, 1999). The consequence of this view is the principle of compositionality. The idea that the meaning of a sentence is the sum of (i) the meanings of the individual parts of the sentence (e.g. the words) and (ii) the way in which those parts are combined.

This view breaks down when confronted with noncompositional expressions. The meaning of idioms such as kick the bucket, and phrasal verbs such as chew out, cannot be interpreted by relying upon their structure and the meaning of their individual components.

Early approaches incorporated these expressions into the lexicon by treating them as word-like units (Bobrow \& Bell, 1973; Swinney \& Cutler, 1979), a hypothesis we call the Lexical Representation Hypothesis (LRH). Under the $\mathrm{LRH}$, the representation of an idiomatic expression such as kick the bucket is similar or identical to the representation of a single word. The idiom is stored as a single unit linked to whatever idiosyncratic meaning it has, and processing of the idiom proceeds in the same manner as any other lexical unit. It is worth noting that in many idioms are ambiguous (i.e. kick the bucket: to die or to kick the pail), but this is not unique to idioms. Many words are also ambiguous (e.g. bank). Under the LRH the mechanisms for dealing with word ambiguity also apply to idioms.

This avenue of research was responsible for many of the seminal findings regarding idiom processing. It was discovered that prior context can influence the interpretation of a sentence with a literal/non-literal ambiguity (Bobrow \& Bell, 1973), and also that idiomatic expressions are generally recognized and processed more rapidly than literal expressions (Swinney \& Cutler, 1979; Cacciari et al. 2007). These discoveries were taken as evidence for the LRH, as they suggested different processing modes for literal and non-literal expressions, and an inattention to the syntactic structure of idioms.

However, more recent work has raised doubts concerning the LRH. Tabossi et al. (2009) demonstrated that the apparent non-literal advantage in processing speed is due to frequency of usage rather than non-literalness, and extends to the greater class of fixed expressions (e.g. clichés). Other work has demonstrated that the parser is sensitive to the syntactic structure of idiomatic expressions (Peterson et al. 2001, Cacciari et al 1988), a finding not predicted by the LRH.

Several contemporary models of now adopt a Structural Representation Hypothesis (SRH), in which access to the idiomatic representation of an expression is mediated by the literal components of the expression (Sprenger et al. 2006; Cutting \& Bock 1997; Gibbs \& Nayak 1989; Tabossi et al. 2009).

\subsection{Aims of this Research}

While previous research has investigated the general properties of idiom processing, and to a lesser degree how comprehenders decide upon an interpretation when faced with a literal/non-literal ambiguity, the nature and the time-course of the idiom comprehension process - in particular, how comprehenders recover from violated expectations concerning (non)1iteralness - is not yet fully understood. Our study investigates what happens when a comprehender's ini- 
Bias

(a)

The foolish entrepreneur,

(b)

The daring fireman,

who liked living on the edge,

who liked living on the edge,

who liked living on the edge,

(c)

(d)

\section{Verb+P}

rushed into

rushed into

rushed into

rushed into

\section{Resolution}

the building

the building

the decision

the decision
Spillover

without hesitation last Saturday

without hesitation last Saturday

without hesitation last Saturday

without hesitation last Saturday

\section{Key: Literal Non-Literal}

Figure 1- Example stimuli. Target items differed by condition only in the content of the Bias and Resolution regions. Text in red italics is for the non-literal conditions while text in blue bold is for the literal conditions.

tial preferred interpretation of a literal/non-literal ambiguity is later revealed to be incorrect.

\subsection{Predictions}

The LRH predicts that processing will be easier when the comprehender's initial bias is towards a literal interpretation and this expectation has to be adjusted in favor of a non-literal interpretation. In other words, if you expect a literal interpretation but the expression turns out to be used in the non-literal way, processing will proceed more smoothly than if you were expecting a non-literal interpretation in a situation that in the end turns out to involve a literal interpretation This is because, under the LRH, shifting to a non-literal interpretation is as easy as looking up an individual word; no explicit structure building needs to take place.

According to SRH-based models, the retrieval of literal meaning is obligatorily. Thus, regardless of whether the comprehender is expecting a literal or non-literal interpretation, it might be that dealing with an expression that turns out to have a literal interpretation is always easy. Depending on the model this may arise as either a literal advantage or as no advantage, however, none of these models predict a non-literal advantage.

\section{Method \& Procedure}

Twenty-four English speakers read sentences displayed on a computer monitor and answered simple questions about the sentences.

\subsection{Stimuli}

The experimental stimuli consisted of 16 targets and 32 fillers.

As shown in Fig.1, each target involves an ambiguous verb $+p$ sequence (e.g. rushed into, dug into, eased off, dwelt on). This sequence is preceded by a biasing region, which creates a bias toward either a literal $(b, d)$ or non-literal $(a, c)$ interpretation of the ambiguous verb $+p$ sequence.
We also manipulated whether the sentence disambiguated towards a literal or non-literal interpretation. This disambiguation occurs in the resolution region, which resolved the verb $+p$ sequence to a literal $(a, b)$ or non-literal $(\mathrm{c}, \mathrm{d})$ interpretation. These two factors, bias direction and resolution direction were crossed to create four conditions, as can be seen in Fig.1. Two of these conditions are Congruent (resolution matches expectation from bias, b,c); two are Incongruent (resolution does not match bias, a,d)

The stimuli also contained a lead-in region and a spillover region. The lead-in region served as a buffer between the bias and the verb $+p$ sequence, and the spillover region served as a buffer region between the resolution and the end of the sentence. Within each target item the verb $+p$, lead-in, and spillover were identical, and each participant saw each target item in only one condition.

\subsection{Norming}

Stimuli were normed/pre-tested in a series of experiments to ensure that the information in the biasing region biased comprehenders in the intended direction, and that the verb $+p$ sequences were sufficiently ambiguous. These studies also provided (i) a quantitative metric of biasing region strength and (ii) a baseline measure of the lexical bias of each verb $+p$ sequence (i.e. how strongly biased it is towards a literal or non-literal interpretation in the absence of any context). These measures were used in later analyses to help remove possible verb-specific or item-specific effects that could mask the effects of (non)literalness that we are interested in.

\section{4.}

Procedure

Participants completed a standard self-paced reading task. Each sentence was initially presented with all letters replaced by hyphens. Participants pressed a key to unmask the first word and then pressed the key again yo re-mask the first word and unmask the second word, and so on. Participants were instructed to 


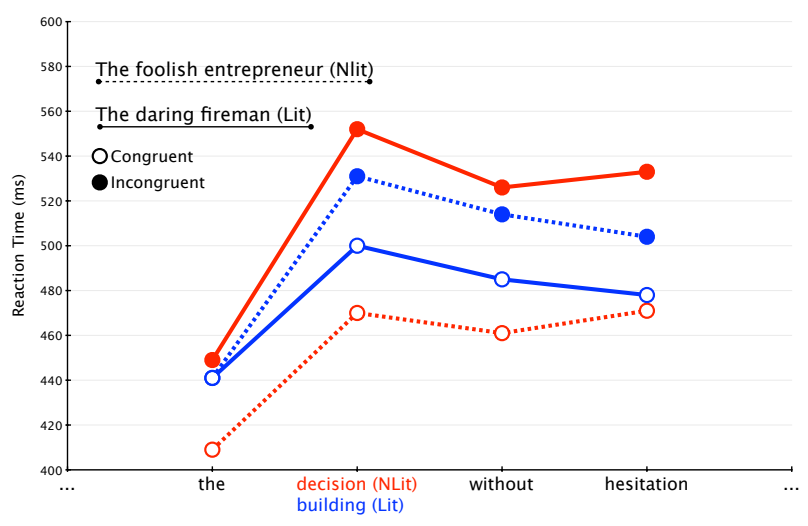

Figure 2- Reaction times over the region of interest.

read sentences at their own pace, but to ensure that they fully understood each sentence.

Occasionally participants answered a yes/no question about the preceding sentence.

The study was run with E-Prime (Psychology Software Tools, Inc).

\section{Analyses}

Analyses were performed over log-transformed reaction times (RTs). ${ }^{1}$ RTs were averaged over the four-word region following the verb, consisting of the resolution and the first two words of the spillover region (e.g. the building without besitation), to capture the resolution and to provide a brief spillover region ${ }^{2}$. This average was analyzed using a linear mixed effects model (Baayen, 2008) with Congruency and Bias Direction as fixed effects and Items and Subjects as random effects. We also included the bias strength for each biasing region and the lexical bias of each $v e r b+p$ sequence from the norming studies as fixed effects.

6.

Analyses revealed a significant main effect of bias direction $(\beta=0.05, \mathrm{t}=2.28, \mathrm{p}<.05)$ and Congruency $(\beta=0.07, t=4.1, p<.001)$ but no interaction. Thus, as can be seen in Fig.2, congruent conditions are gener-

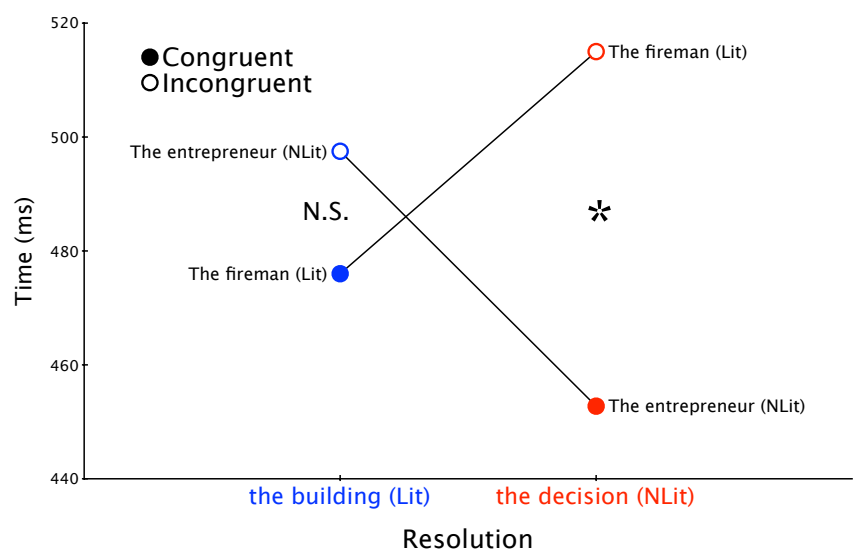

Figure 3- Plot of averaged reaction times.

ally faster than incongruent conditions and non-literal biased conditions are typically faster ${ }^{3}$

In order to take a closer look at how fulfilled vs violated expectations influence the processing of (non)literal interpretations, we compared the two literal-resolving conditions ( $a, b)$ to each other, as well as the two non-literal-resolving conditions (c,d). Paired t-tests revealed a significant difference between the two non-literal resolving conditions $(\mathrm{t}(23)=-3.15, \mathrm{p}<.01)$ but no significant difference between the literal resolving conditions $(\mathrm{t}(23)=.82$, $\mathrm{p}=.4$ ) see Fig.3. In other words, when the verb $+p$ sequence is disambiguated towards a literal interpretation (e.g. ...the building), RTs are equally fast regardless of whether participants were expecting a literal or non-literal interpretation. But when the disambiguation region (e.g. ...the decision) signals a nonliteral interpretation, participants pay a penalty (slow down) when they were expecting a literal interpretation.

7.

Discussion

As we saw in Fig.3, contextual expectations play a crucial role when the ambiguity is resolved to a nonliteral interpretation, but not when the ambiguity is resolved literally. These results suggest that when the parser encounters an unexpected interpretation, it is more readily able to recover when its incorrect inter-

\footnotetext{
${ }^{1}$ Analyses were also performed on untransformed data, and the same pattern of results emerged.

2 A four-word region was chosen as it included the disambiguating region and the first content word following the disambiguating region.
}

3 We also found that subject differences $(\chi 2=328.5 \mathrm{p}<.001)$, item differences $(\chi 2=13.44, \mathrm{p}<.001)$ and biasing region strength $(\chi 2=5.9, \mathrm{p}<.05)$ contributed significantly to model fit, but found no significant effects of lexical bias. 
pretation is toward a literal meaning rather than a non-literal one. There are several reasons why this may be the case $e^{4}$.

First, it might be that the parser can more rapidly access literal meaning during recovery processes. Thus the apparent lack of context effects in the case of a literal resolution is a result of the parser's ability to quickly access the literal meaning. With non-literal resolution, however, the parser is more severely impacted by incongruent bias, as recovering the nonliteral meaning takes longer.

A second possibility is that the parser obligatorily considers the literal meaning regardless of bias. Several models explicitly predict non-literal interpretations are accessed via the lexical representation of the literal components. Thus, the lack of context effects in the literal resolution condition could be a side-effect of this obligatory literal processing.

The present experiment was not designed to decide between these two alternatives, as its main aim was to probe context effects on real-time idiom processing more generally. However research into idiom processing has generally revealed a pervasive processing advantage for idioms. That is, all else being equal, idioms tend to be processed more rapidly than matched literal expressions - a finding which would be hard to explain with the former option.

Regardless of the underlying cause, our results clearly support the SRH over the LRH. There appears to be a clear literal advantage in recovery processes, a fact not easily accounted for by the LRH. Additionally, these results suggest that SRH models which predict free spreading activation between the structural/iteral representation and the idiomatic representation are incomplete, since access to the non-literal interpretation does not appear to be obligatory.

One interesting possibility is that contextual information is used to actively suppress interpretations which are expected to be low probability. Given that the representation of the non-literal meaning is essentially word like, while the literal representation of the same string is distributed over several syntactic nodes, active inhibition could explain why on the one hand, literal meaning seems obligatory even in improbable contexts (i.e. as it is distributed, it is harder to suppress), and still capture the pervasive finding that non-literal retrieval is more rapid (i.e as it is not distributed, there are less operations required to retrieve it). This dual-representation with inhibition hypothesis will require further investigation, however.

\section{8.}

\section{References}

Baayen, H. (2008). Analyzing linguistic data: A practical introduction to statistics using R. Cambridge: CUP.

Bobrow, S. A. \& Bell, S. M. (1973). On catching on to idiomatic expressions. Memory \& Cognition, 1 , 343-46.

Cacciari, C., Padovani, R. \& Corradini, P. (2007). Exploring the relationship between individuals' speed of processing and their comprehension of spoken idioms. European Journal of Cognitive Psychology, 19, 417-45.

Cutting, J. C., \& Bock, K. (1997). That's the way the cookie bounces: Syntactic and semantic components of experimentally elicited idiom blends. Memory \& Cognition, 25, 57-71.

Dell, G. (1986). A spreading activation theory of retrieval in language production. Psychological Review, 93, 283-321.

Forster, K. (1976). Accessing the mental lexicon. In R. Wales \& E. Walker (Eds.), New Approaches to Language Mechanisms. (257-287) Amsterdam: North Holland.

Gibbs W.R. \& Nayak, N. P. (1989). Psycholinguistic studies on the syntactic behavior of idioms. Cognitive Psychology, 21, 100-38.

Levelt, W. J. M. (1999). Models of word production. Trends in Cognitive Sciences, 3, 223-232.

Peterson, R. R., Burgess, C., Dell, G. S. \& Eberhard K. L. (2001). Dissociation between syntactic and semantic processing during idiom comprehension. Journal of Experimental Psychology: Learning, Memory and Cognition, 90, 227-34.

Sprenger S. A., Levelt, W. J. M. \& Kempen, G. (2006). Lexical access during the production of idiomatic phrases. Journal of Memory and Language, 54, $161-184$

Swinney \& Cutler. (1979). The access and processing of idiomatic expressions. Journal of Verbal Learning \& Verbal Behaviour, 18, 523-34.

Tabossi, P., Fanari, R. \& Wolf, K. (2009). Why are idioms recognized fast? Memory \& Cognition, 37 , $529-40$

4 We remain agnostic as to whether the observed context effects are due to the active revision of a misparse or to the parser needing to re-weight/re-rank some array of possible parses. The crucial finding is that the recovery process, whatever that entails, is asymmetric 